\title{
Corneal confocal microscopy: a non-invasive surrogate of nerve fibre damage and repair in diabetic patients
}

\author{
R. A. Malik ${ }^{1}$, P. Kallinikos ${ }^{2}$, C.A. Abbott ${ }^{1}$, C.H.M. van Schie ${ }^{1}$, P. Morgan ${ }^{2}$, N. Efron ${ }^{2}$, A. J. M. Boulton ${ }^{1}$ \\ ${ }^{1}$ Department of Medicine, Manchester Royal Infirmary, Manchester, UK \\ ${ }^{2}$ Eurolens Research, Department of Optometry and Neuroscience, University of Manchester Institute of Science and Technology, \\ Manchester, UK
}

\begin{abstract}
Aims/hypothesis. The accurate detection, characterization and quantification of human diabetic neuropathy are important to define at risk patients, anticipate deterioration, and assess new therapies. Corneal confocal microscopy is a reiterative, rapid, non-invasive in vivo clinical examination technique capable of imaging corneal nerve fibres. The aim of this study was to define the ability of this technique to quantify the extent of degeneration and regeneration of corneal nerve fibres in diabetic patients with increasing neuropathic severity.

Methods. We scanned the cornea and collected images of Bowman's layer (containing a rich nerve plexus) from 18 diabetic patients and 18 age-matched control subjects.
\end{abstract}

Diabetic polyneuropathy is a major clinical problem which currently has no effective therapy. The accurate detection, characterization and quantification of this condition are important to define at risk patients, anticipate deterioration, and assess new therapies [1]. Whilst electrophysiology and quantitative sensory tests allow quantification of neuropathic severity, they demonstrate a limited ability to target the fibre types which could benefit from therapeutic intervention, and are unable to demonstrate repair $[2,3]$. We have re-

Received: 13 November 2002 / Revised: 23 December 2002 Published online: 9 May 2003

CC Springer-Verlag 2003

Corresponding author: Dr. R. A. Malik, Department of Medicine, Manchester Royal Infirmary, Oxford Road, Manchester, M13 9WL, UK

E-mail: Rayaz.A.Malik@man.ac.uk
Results. Corneal nerve fibre density $\left(\mathrm{F}_{3}=9.6, p<0.0001\right)$, length $\left(\mathrm{F}_{3}=23.8, \quad p<0.0001\right)$, and branch density $\left(\mathrm{F}_{3}=13.9, p<0.0001\right)$ were reduced in diabetic patients compared with control subjects, with a tendency for greater reduction in these measures with increasing severity of neuropathy.

Conclusion/Interpretation. Corneal confocal microscopy is a rapid, non-invasive in vivo clinical examination technique which accurately defines the extent of corneal nerve damage and repair and acts as a surrogate measure of somatic neuropathy in diabetic patients. It could represent an advance to define the severity of neuropathy and expedite assessment of therapeutic efficacy in clinical trials of human diabetic neuropathy. [Diabetologia (2003) 46:683-688]

Keywords Diabetic neuropathy, confocal microscope, corneal nerves, surrogate, degeneration, regeneration.

cently used corneal confocal microscopy, to define A $\delta$ and $\mathrm{C}$ fibre structure in normal subjects [4].

We propose that this technique could be useful to define nerve fibre damage and repair in diabetic patients for two major reasons. Corneal innervation provides protective and trophic functions in corneal repair in relation to disease, trauma or surgery. This could be relevant as a recent study has shown a higher risk of developing post-operative epithelial complications and poorer refractive results in diabetic patients undergoing laser-assisted in situ keratomileusis (LASIK) for myopia [5]. Of greater clinical and therapeutic relevance, we propose that this technique will allow a rapid, in vivo, and non-invasive means to accurately demonstrate nerve fibre damage and repair.

In a recent interventional study with an aldose reductase inhibitor, paired sural nerve biopsies showed regenerative activity confined almost exclusively to 
small myelinated fibres [6], which cannot be assessed using electrophysiology [2]. Whilst quantitative sensory tests of thermal and pain perception have been proposed to assess such small fibre damage $[2,3]$, we have recently shown no relationship between quantitative sensory tests and small myelinated or unmyelinated fibre damage and repair [7]. This might explain the disappointing results of clinical trials with recombinant nerve growth factor ( $\mathrm{rNGF}$ ), which targets small fibres [8]. Similarly, a recent study of brain-derived neurotrophic factor (rhBDNF) also failed to establish efficacy as the two measures employed to establish efficacy (cold perception threshold and hair follicle innervation) were often absent in many of the patients in the study [9]. Sural nerve biopsy with electron microscopy $[6,7]$, and ex vivo confocal microscopy of skin-punch or skin blister biopsies [10] allow direct examination of thinly myelinated and unmyelinated nerve fibre damage and repair. However, both are invasive procedures, and the functional relevance of the morphological changes is not clearly defined. This study was designed to assess the ability of corneal confocal microscopy to define corneal sensory nerve structure and to develop morphological markers of nerve damage and repair in diabetic patients in relation to the severity of somatic diabetic neuropathy.

\section{Subjects and methods}

Patients. Eighteen diabetic patients underwent evaluation of neuropathic severity and corneal confocal microscopic examination. Patients with any other known cause of neuropathy or previous corneal abnormality were excluded. Confocal microscopy was carried out on a further 18 age-matched control subjects. The protocol used was approved by the local research ethics committee of the Greater Manchester Health Authority and all patients gave written informed consent.

Evaluation of neuropathic severity. All patients underwent a detailed clinical history and examination to rule out any other cause of neuropathy. The neuropathy disability score (NDS) was based on a clinical scoring system obtained from a neurological examination which defined abnormalities of vibration perception using a tuning fork, pin prick perception and temperature perception as well as the presence or absence of ankle reflexes, producing a score ranging from 0-10 [11, 12]. Quantitative vibration and heat pain assessment were carried out with the Computer Aided Sensory Evaluator IV (CASE IV) (WR Medical Electronics, Minn., USA) [13]. An MS92a EMG machine (Medelec Limited, Old Woking, Surrey, UK) was used to determine the peroneal motor nerve conduction velocity (PMNCV) $(\mathrm{m} / \mathrm{s})$.

Based on the above evaluations, patients were stratified into those with mild (NDS $<3, \mathrm{VPT}<25, \mathrm{PMNCV}>35$ ), moderate (NDS-3-6, VPT 25-35, PMNCV-30-35) and severe (NDS $>6$, VPT $>35$, PMNCV<30) neuropathy.

Confocal microscopy. Patients underwent examination with a Tomey Confoscan corneal confocal microscope Model P4 (Erlangen, Germany). Each eye being examined was anaesthetised with one drop of benoxinate hydrochloride $0.4 \%$ (oxybuprocaine hydrochloride, Minims). The objective lens of the confocal microscope was disinfected (isopropyl alcohol $70 \% \mathrm{v} / \mathrm{v}$, Swabs). A large drop of Viscotears liquid gel (carbomer 940, Ciba Vision Ophthalmics) was applied onto the tip of the lens and advanced slowly forwards until the gel touched the cornea, allowing optical but not physical contact between the objective lens and corneal epithelium during the examination.

One eye of each subject was selected at random for examination. Several scans of the entire depth of the cornea were recorded by turning the fine focus of the objective lens backwards and forwards for approximately 2 min to acquire satisfactory images of all corneal layers providing en face two dimensional images with a lateral resolution of approximately 1 to $2 \mu \mathrm{m}$ and final image size of 768 pixels $\times 576$ pixels. On average three to five high quality images of Bowman's layer were available for investigation in all patients and control subjects. This layer is of particular relevance for defining neuropathic changes since it is the location of the main nerve plexus that supplies the overlying corneal epithelium. The investigator who examined the cornea with the confocal microscope and who undertook morphometric measurements of the images of Bowman's layer was masked with respect to the severity of neuropathy of the diabetic patients.

Using the images collected we have developed the following three parameters as potential indicators of corneal nerve fibre damage and repair: (i) Nerve fibre density (NFD) - the total number of major nerves per square millimetre of corneal tissue; (ii) Nerve fibre length (NFL) - the total length of all nerve fibres and branches ( $\mathrm{mm}$ per square millimetre) of corneal tissue; (iii) Nerve branch density (NBD) - the number of branches emanating from major nerve trunks per square millimetre of corneal tissue.

Measures (i) and (iii) above were determined using morphometric software incorporated within the Tomey instrument. Measure (ii) was determined using third party image analysis software (Scion Image for Windows, Scion Corporation, Frederick, Ma., USA).

To estimate the error in measuring NFD, NFL and NBD we acquired images and determined each of these parameters on 15 subjects on two occasions separated by at least $48 \mathrm{~h}$. The coefficient of variation of these parameters was determined to be: $12 \%$ for NFD, $9 \%$ for NFL and $24 \%$ for NBD.

Statistical methods. A multivariate analysis of variance (MANOVA) was conducted to compare the three measures of confocal microscopic nerve appearance for the four groups (mild, moderate and severe neuropathy patient groups, and the control subjects). Where differences within the clinical groups were established at the $p=0.05$ level, post-hoc analysis was conducted using the Tukey Kramer test and expressed as an $\mathrm{F}$ value for three degrees of freedom $\left(\mathrm{F}_{3}\right)$ supported with a defined $p$ value. A $p$ value of less than 0.05 was considered statistically significant.

\section{Results}

Whilst age, duration of diabetes and $\mathrm{HbA}_{1 \mathrm{c}}$ were greatest in patients with the most severe neuropathy this was not different between groups (Table 1). Based on our pre-defined criteria of clinical severity, quantitative sensory tests and electrophysiology, four patients were classified as 'mild', seven as 'moderate' and seven as 'severe' (Table 2). 


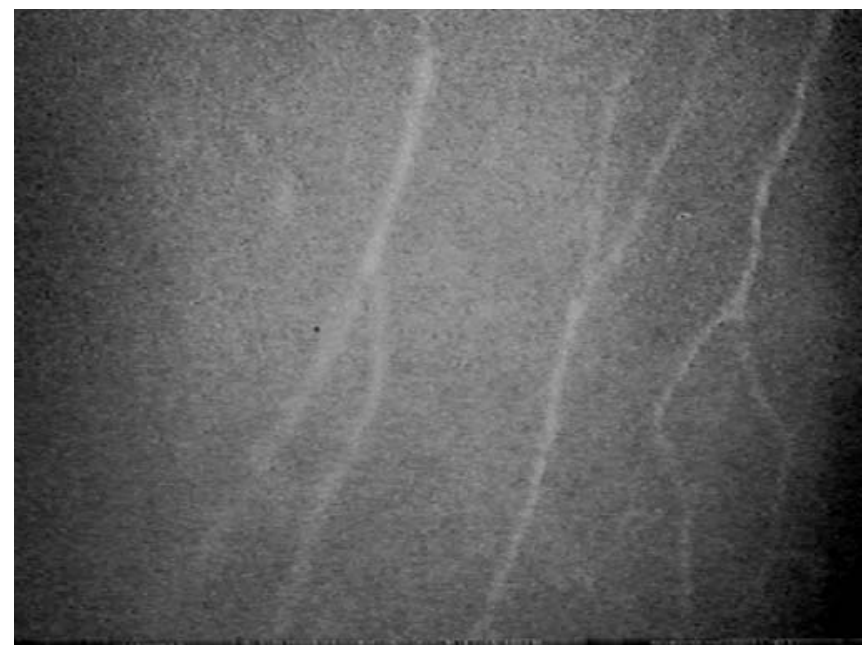

Fig. 1. Bowman's layer of the cornea of a normal (control) subject

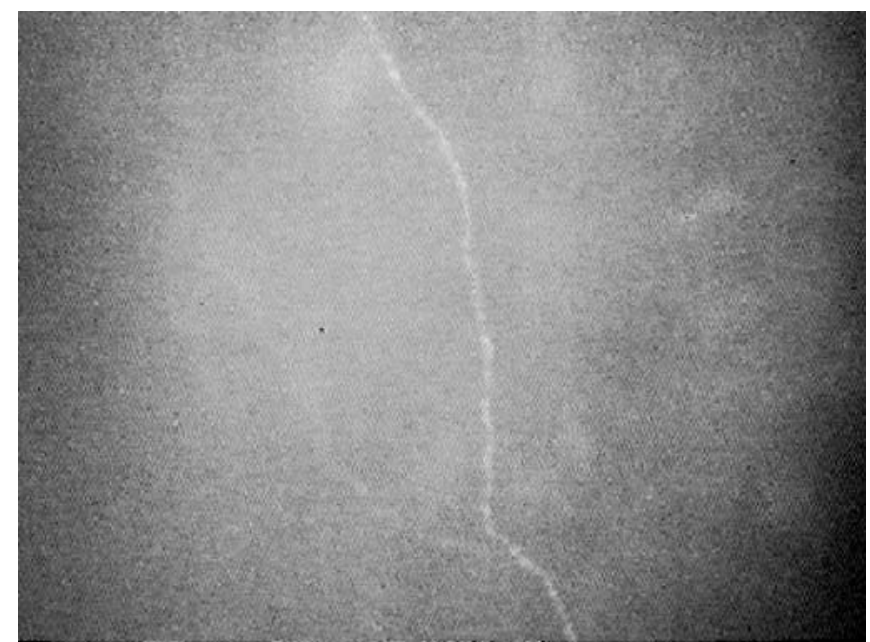

Fig. 2. Bowman's layer of the cornea of a patient with severe diabetic neuropathy

Table 1. Clinical details (mean \pm standard deviation)

\begin{tabular}{lllll}
\hline Parameter & Control $(n=18)$ & Mild $(n=4)$ & Moderate $(n=7)$ & Severe $(n=7)$ \\
\hline Age (yrs.) & $57.8 \pm 11.5$ & $53.0 \pm 18.5$ & $60.1 \pm 7.4$ & $58.3 \pm 12.4$ \\
Diabetes duration (yrs.) & 0 & $21.3 \pm 3.6$ & $20.8 \pm 5.1$ & $26.0 \pm 7.4$ \\
Diabetes (Type 1/Type 2) & $<6.5$ & $2 / 2$ & $2 / 5$ & $3 / 4$ \\
HbA $_{1 \mathrm{c}}(\%)$ & $<$ & $7.8 \pm 0.8$ & $8.1 \pm 1.2$ & $8.2 \pm 1.4$ \\
\hline
\end{tabular}

Table 2. Data from the neuropathic evaluation tests. Neuropathy deficit score (NDS), peroneal motor nerve conduction velocity (PMNCV), vibration perception threshold (VPT), heat pain assessment (HPA) (mean \pm standard deviation), just noticeable difference (JND)

\begin{tabular}{|c|c|c|c|c|}
\hline Parameter & Control $(n=18)$ & Mild ( $n=4)$ & Moderate $(n=7)$ & Severe $(n=7)$ \\
\hline NDS & 0 & $1.2 \pm 0.6$ & $3.5 \pm 0.9$ & $7.5 \pm 1.2$ \\
\hline $\operatorname{PMNCV}\left(\mathrm{ms}^{-1}\right)$ & $>45$ & $37.6 \pm 3.4$ & $33.5 \pm 4.2$ & $26.2 \pm 4.5$ \\
\hline TPT (JND) & $<15$ & $17.6 \pm 2.2$ & $23.8 \pm 1.1$ & $>25.0$ \\
\hline
\end{tabular}

Table 3. Data from the confocal microscope evaluation of the nerve fibres in Bowman's layer of the cornea. Nerve fibre density (NFD), nerve fibre length (NFL), nerve branch density (NBD) (mean \pm standard deviation)

\begin{tabular}{|c|c|c|c|c|}
\hline Parameter & Control $(n=18)$ & Mild $(n=4)$ & Moderate $(n=7)$ & Severe $(n=7)$ \\
\hline NFD (number/mm²) & $44.5 \pm 14.1$ & $37.2 \pm 4.6$ & $24.4 \pm 5.5$ & $21.2 \pm 9.4$ \\
\hline $\mathrm{NFL}\left(\mathrm{mm} / \mathrm{mm}^{2}\right)$ & $13.5 \pm 0.3$ & $10.8 \pm 0.9$ & $7.5 \pm 1.1$ & $4.3 \pm 1.5$ \\
\hline
\end{tabular}

Figure 1 shows an image of corneal nerves in Bowman's layer, showing three fibres with typical beaded appearance, mild tortuosity, and adequate branching. Figure 2 shows a typical image from a diabetic patient with severe neuropathy. Only one nerve fibre is evident, with a single branch leaving the main nerve trunk at the bottom of the frame.

The data for the three morphometric variables are given in Table 3.
For NFD, there were statistical differences between the four groups $\left(\mathrm{F}_{3}=9.6, p<0.0001\right)$. NFD was reduced in the moderate $(p<0.01)$ and severe $(p<0.01)$ groups compared with the control group (Fig. 3).

For NFL, there were statistical differences between the four groups $\left(\mathrm{F}_{3}=23.8, p<0.0001\right)$. NFL was reduced in the moderate $(p<0.01)$ and severe $(p<0.01)$ groups compared with the control group. Furthermore, NFL was lower in the severe group compared with the mild group (Fig. 4). 


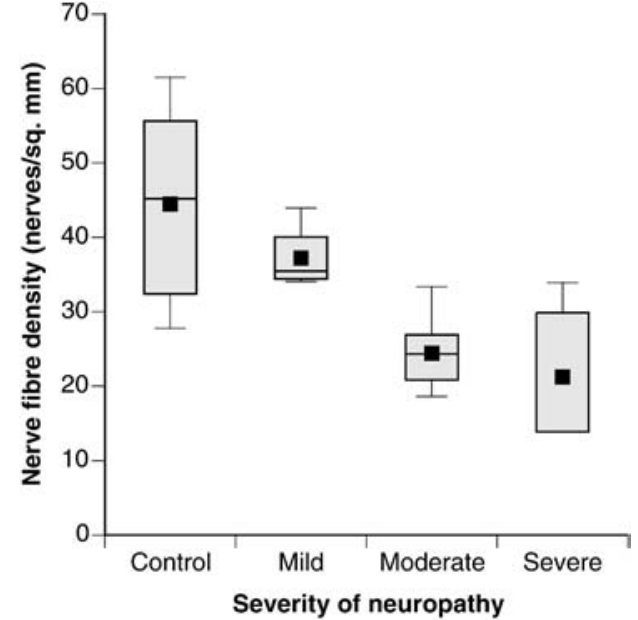

Fig. 3. Nerve fibre density in diabetic patients and control subjects. The solid black square is the mean of the data set. The horizontal line within the shaded box represents the median of the data set. The upper and lower limits of the shaded box indicate the 75 th and 25 th centiles, respectively. The upper and lower limits of the whiskers indicate the 90th and 10th centiles, respectively

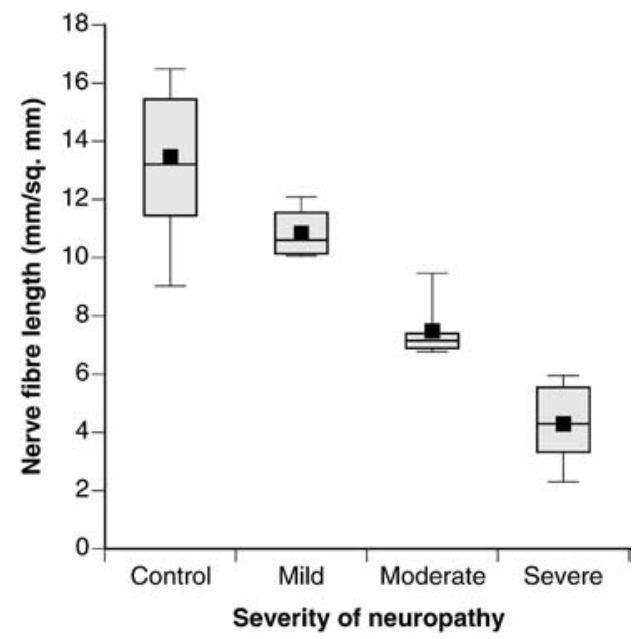

Fig. 4. Nerve fibre length in diabetic patients and control subjects. Description of the statistics shown in the box and whisker plots

There were statistical differences between the four groups for $\mathrm{NBD}\left(\mathrm{F}_{3}=13.9, p<0.0001\right)$. NBD was reduced in all three diabetic groups $(p<0.01)$ compared with the control group (Fig. 5).

\section{Discussion}

The cornea is the most densely innervated part of the human body deriving its innervation by the ophthalmic division of the trigeminal nerve, via the anterior ciliary nerves. Corneal nerves have been studied in detail by light and electron microscopy $[14,15,16]$, in combination with immunohistochemical techniques

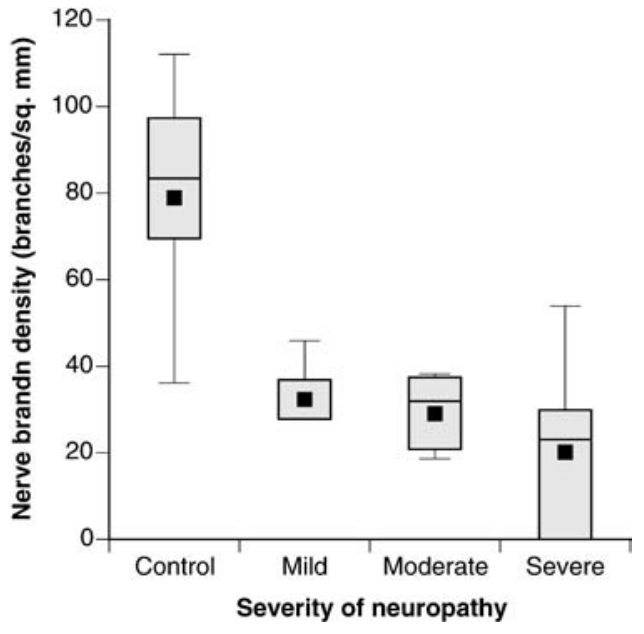

Fig. 5. Nerve branch density in diabetic patients and control subjects. Description of the statistics shown in the box and whisker plots

$[17,18,19,20]$ to define their distribution, ultrastructural anatomy and neurotransmitter types.

The recent application of confocal microscopy to image the cornea provides a new approach to the study of corneal nerve morphology [21]. It provides a rapid in vivo, non-invasive technique which enables prospective and reiterative evaluation of the human cornea at $700 \times$ magnification. Two recent studies including our own have quantified corneal nerve morphology in healthy subjects [4 21, 22] and shown changes with ageing [22].

It could be argued that corneal innervation has little clinical relevance to the cornea and limited application as a measure of somatic neuropathy in diabetic patients. However, corneal sensation is impaired within 3 months in both diabetic [23] and galactose-fed [24] rats. Moreover, sub-clinical abnormalities of corneal innervation [25] and corneal ulceration are a recognized feature in diabetic patients [26]. Corneal aesthesiometry and confocal microscopy have shown a reduction in both corneal sensitivity and the number of corneal nerve fibre bundles, in patients with Type 1 diabetes [27]. Furthermore, the number of corneal nerve fibres correlated with the severity of somatic neuropathy evaluated from the Michigan Neuropathy Screening Instrument [27]. In a patient with corneal dystrophy secondary to corneal denervation from hereditary sensori-motor neuropathy a sural nerve biopsy showed small myelinated fibre damage with sparing of the large fibres. This shows the close association between the extent and type of nerve damage in a peripheral nerve and the cornea [28]. Of therapeutic relevance, loss of corneal sensitivity could be ameliorated by topical application of an aldose reductase inhibitor in both animals $[23,24]$ and in diabetic patients [29].

Ocular surgery, in particular photorefractive keratectomy, results in corneal nerve damage and loss of 
corneal sensitivity for up to 1 year [30]. Corneal epithelial metabolism, cell adhesion and wound healing depend on adequate corneal innervation [31]. Thus a recent study has shown a higher risk of developing postoperative epithelial complications and poorer refractive results in diabetic patients undergoing LASIK [5].

Topical application of NGF to the cornea and presumed restoration of corneal innervation has shown remarkable rates of healing in patients with recalcitrant neurotrophic corneal ulcers [32]. Similarly, in a patient with neurotrophic keratopathy who underwent topical treatment with a substance-P derived peptide and IGF-1, confocal microscopy showed regeneration of corneal nerve fibres within 4 months [33]. This approach offers a means of reducing the time window within which therapeutic efficacy of candidate drugs can be established, as healing of corneal ulcers secondary to corneal reinnervation began within 10 days [32].

As corneal nerve integrity depends on nutrient diffusion from the basal endothelial layer as opposed to capillaries it will be influenced to a lesser extent by concomitant microangiopathy, which clearly plays a major role in somatic nerves [34]. However, a recent study using confocal biomicroscopy has shown increased corneal basement membrane abnormality which related to the severity of retinopathy in diabetic patients [35]. This suggests that basement membrane thickening, the hallmark of diabetic microangiopathy also occurs in the cornea and might be relevant to the integrity of the corneal nerves.

To date the assessment of corneal nerve fibre morphology with confocal microscopy has been rather basic and limited for the purposes of interpreting small fibre degeneration and regeneration [27]. However, recent studies using confocal microscopy in normal subjects has refined and improved the quantification of $\mathrm{C}$ and $A \delta$ corneal nerve fibres $[4,22]$. These values are in high concordance with previous histological studies $[14,15,16]$.

This study has shown a progressive reduction in the number of corneal nerve fibres in diabetic patients, suggestive of enhanced degeneration. We have also shown a reduction in the number of corneal nerve branches, suggestive of a reduction in regenerative capacity with progression of neuropathic severity. It is interesting to observe that the technique is sensitive enough to detect an abnormality in the corneal nerves of patients deemed to have mild neuropathy using conventional tests.

Therefore, corneal confocal microscopy offers a rapid, non-invasive, and reiterative technique to quantify corneal nerve fibre degeneration and potentially regeneration. It provides an accurate means of detection, characterization and quantification of nerve damage in diabetic patients and provides a means to assess therapeutic efficacy of treatments in clinical trials of human diabetic neuropathy. Moreover, because therapies can be applied topically to the cornea, nerve fibre regeneration can be assessed directly with confocal microscopy. This will enable rapid assessment of drug efficacy and expedite drug development programmes for therapies deemed to be beneficial for somatic human diabetic neuropathy.

Acknowledgements. This work was supported by an Innovative grant from Juvenile Diabetes Research Foundation International.

\section{References}

1. Boulton AJM, Malik RA (1998) Diabetic neuropathy. Med Clin North Am 82:909-929

2. Dyck PJ, O'Brien PC, Litchy WJ, Harper CM, Daube JR, Dyck PJ (2001) Use of percentiles and normal deviates to express nerve conduction and other test abnormalities. Muscle Nerve 24:307-310

3. Arezzo JC (1999) New developments in the diagnosis of diabetic neuropathy. Am J Med 30:9S-16S

4. Oliveira-Soto L, Efron N (2001) Morphology of corneal nerves using confocal microscopy. Cornea 20:374-384

5. Fraunfelder FW, Rich LF(2002) Laser-assisted in situ keratomileusis complications in diabetes mellitus. Cornea 21: 246-248

6. Greene DA, Arezzo JC, Brown MB (1999) Effect of aldose reductase inhibition on nerve conduction and morphometry in diabetic neuropathy. Zenarestat Study Group. Neurology 53:580-591

7. Malik RA, Veves A, Walker D et al. (2001) Sural nerve fibre pathology in diabetic patients with mild neuropathy: relationship to pain, quantitative sensory testing and peripheral nerve electrophysiology. Acta Neuropathol (Berl) 101:367-374

8. Apfel SC, Schwartz S, Adornato BT et al. (2000) Efficacy and safety of recombinant human nerve growth factor in patients with diabetic polyneuropathy: A randomized controlled trial. JAMA 284:2215-2221

9. Wellmer A, Misra VP, Sharief MK, Kopelman PG, Anand P (2001) A double-blind placebo-controlled clinical trial of recombinant human brain-derived neurotrophic factor (rhBDNF) in diabetic polyneuropathy. J Peripher Nerv Syst 6:204-210

10. Holland NR, Stocks A, Hauer P, Cornblath DR, Griffin JW, McArthur JC (1997) Intraepidermal nerve fibre density in patients with painful sensory neuropathy. Neurology 48:708-711

11. Young MJ, Boulton AJM, Macleod AF, Williams DRR, Sonksen PH (1993) A multicentre study of the prevalence of diabetic peripheral neuropathy in the United Kingdom hospital clinic population. Diabetologia 36:150-154

12. Abbott CA, Carrington AL, Ashe H et al. (2002) The NorthWest Diabetes Foot Care Study: incidence of, and risk factors for, new diabetic foot ulceration in a community-based patient cohort. Diabet Med 19:377-384

13. Dyck PJ, Zimmerman IR, Johnson DM et al. (1996) A standard test of heat-pain responses using CASE IV. J Neurol Sci 136:54-63

14. Ueda S, Del Cerro M, Rao GN, Aquavella JV (1988) The epithelial innervation in human cornea. A light and electron microscopic study. In: Cavanagh D (ed.) The Cornea: transactions of the World Congress on the Cornea III. Raven Press, New York, pp 63-67 
15. Müller LJ, Pels L, Vrensen GFJM (1996) Ultrastructural organisation of human corneal nerves. Invest Ophthalmol Vis Sci 37:476-488

16. Müller LJ, Vrensen GFJM, Pels L, Nunes Cardozo B, Willekens B (1997) Architecture of human corneal nerves. Invest Ophthalmol Vis Sci 38:985-994

17. Tervo T (1977) Consecutive demonstration of nerves containing catecholamine and acetylcholinesterase in the rat cornea. Histochemistry 50:291-299

18. Tervo K, Tervo T, Eränkö L, Vannas A, Cuello AC, Eränkö O (1982) Substance P-immunoreactive nerves in the human cornea and iris. Invest Ophthalmol Vis Sci 23:671-674

19. Tovainen M, Tervo T, Partanen M, Vannas A, Hervonen A (1987) Histochemical demonstration of adrenergic nerves in the stroma of human cornea. Invest Ophthalmol Vis Sci 8:398-400

20. Ueda S, Del Cerro M, Lo Cascio JA, Aquavella JV (1989) Peptidergic and catecholaminergic fibres in the human corneal epithelium. An immunohistochemical and electron microscopic study. Acta Ophthalmol Suppl 192:80-90

21. McGhee CNJ, Keller PR (1998) In vivo confocal microscopy of living tissue: the cornea at cellular level. Eyenews 5:14-20

22. Grupcheva CN, Wong T, Filey AF, McGhee CNJ (2002) Assessing the sub-basal nerve plexus of the living healthy human cornea by in vivo confocal microscopy. Clin and Exp Ophthalmol 30:187-190

23. Hosotani H, Ohashi Y, Kinoshita S, Matsumoto T, Awata T (1996) Effects of topical aldose reductase inhibitor CT-112 on corneal sensitivity of diabetic rats. Curr Eye Res 15:1005-1007

24. Jacot JL, Hosotani H, Glover JP, Lois N, Robison WG (1998) Diabetic-like corneal sensitivity loss in galactose-fed rats ameliorated with aldose reductase inhibitors. J Ocul Pharmacol Ther 14:169-180

25. Urban PP, Forst T, Lenfers M, Koehler J, Connemann BJ, Beyer J (1999) Incidence of subclinical trigeminal and facial nerve involvement in diabetes mellitus. Electromyogr Clin Neurophysiol 39:267-272
26. Hyndiuk RA, Kazarian EL, Schultz RO, Seideman S (1977) Neurotrophic corneal ulcers in diabetes mellitus. Arch Ophthalmol 95:2193-2196

27. Rosenberg ME, Tervo TM, Immonen IJ, Muller LJ, Gronhagen-Riska C, Vesaluoma MH (2000) Corneal structure and sensitivity in type 1 diabetes mellitus. Invest Ophthalmol Vis Sci 41:2915-2921

28. Donaghy M, Hakin RN, Bamford JM et al. (1987) Hereditary sensory neuropathy with neurotrophic keratitis. Description of an autosomal recessive disorder with a selective reduction of small myelinated nerve fibres and a discussion of the classification of the hereditary sensory neuropathies. Brain 110:563-583

29. Hosotani H, Ohashi Y, Yamada M, Tsubota K (1995) Reversal of abnormal corneal epithelial cell morphologic characteristics and reduced corneal sensitivity in diabetic patients by aldose reductase inhibitor, CT-112. Am J Ophthalmol 119:288-294

30. Murphy PJ, Corbett MC, O’Brart DPS, Verma S, Patel S, Marshall J (1999) Loss and recovery of corneal sensitivity following photorefractive keratectomy for myopia. J Refract Surg 15:38-45

31. Beuerman RW, Schimmelpfennig B (1980) Sensory denervation of the rabbit affects epithelial properties. Exp Neurol 69:196-201

32. Lambiase A, Rama P, Bonini S, Caprioglio G, Aloe L (1998) Topical treatment with nerve growth factor for corneal neurotrophic ulcers. N Engl J Med 338:1174-1180

33. Morishige N, Komatsubara T, Chikama T, Nishida $\mathrm{T}$ (1999) Direct observation of corneal nerve fibres in neurotrophic keratopathy by confocal biomicroscopy. Lancet 354:1613-1614

34. Malik RA, Newrick PG, Sharma AK et al. (1989) Microangiopathy in human diabetic neuropathy: relationship between capillary abnormalities and the severity of neuropathy. Diabetologia 32:92-102

35. Morishige N, Chikama TI, Sassa Y, Nishida T (2001) Abnormal light scattering detected by confocal biomicroscopy at the corneal epithelial basement membrane of subjects with type II diabetes. Diabetologia 44:340-345 